\title{
54 GUIDELINES: ARE THEY A WASTE OF TIME?
}

P Saul' ' ${ }^{1} J o h n$ Hunter Hospital, Newcastle, NSW, Australia

10.1136/bmjspcare-2013-000491.54

Background A trickle of guidelines about end of life (EOL) care in the 1990s has become a downpour. Governmental, professional, religious and community groups have released documents ranging from a friendly chat through to standards enforced by government agencies. In response to this deluge, clinicians everywhere have put up their umbrellas.

Methods Evidence for the effectiveness of guidelines is generally poor, and end of life guidance, based on expert opinion, broad in nature, and lacking implementation or audit strategies, fair badly. In addition, the term 'end of life' has no single definition, and no precise tests exist to define to whom EOL guidelines are meant to apply. As a case history, NSW Health has notched up over two decades' experience in writing EOL guidelines, and has attempted to publicise them. But it remains a reality that end of life guidelines can produce a weak justification for change, but cannot themselves produce that change.

Conclusion Improving the management of dying in our institutions is crucial to the credibility of our health systems, and to stem the tide of support for euthanasia. And to achieve this we need a coherent approach with a hierarchy of needs: political will, community pressure, legal support, strategic policy, standard setting, audit, feedback and accountability. Failure to be proactive is sure to lead to pressure of all the wrong kinds, as economic imperatives come to drive urgent efficiencies in end of life care. But an important question remains outstanding: what kind of support do acute care clinicians want (or need?) Without a coherent answer to this empirical question, managing and sustaining change is likely to be beyond us. 